\title{
Nuevas tecnologías y formación permanente del profesorado de educación de adultos
}

\author{
Angel García del Dujo \\ Antonio Víctor Martín García \\ María Dolores Pérez Grande \\ Facultad de Educación. \\ Univ. Salamanca (España)
}

1.- Entre las múltiples aplicaciones de las llamadas Nuevas Tecnologías de la Información y la comunicación se encuentra, sin duda alguna, el campo de la formación permanente; probablemente sea éste el ámbito más inmediato y visible de utilización de una tecnología que entre sus muchas características sobresale su capacidad para superar las dos coordenadas que tradicionalmente han enmarcado y condicionado la acción educativa, el espacio y el tiempo. No es la primera vez, desde luego, que este campo es objeto de reflexión en los Seminarios y Congresos de Teoría de la Educación; por eso, ahora nos vemos exentos de la obligación de razonar y justificar la importancia de la formación permanente de cara al siglo XXI o la inevitabilidad en el presente y en el futuro de acudir a las nuevas tecnologías para llevar a cabo su desarrollo.

Nuestra intención ahora no es, pues, abordar una nueva temática o tendencia a considerar en la educación del nuevo milenio -esto lo damos ya por supuesto-, sino presentar un programa de investigación y desarrollo como consecuencia de la utilización de las nuevas tecnologías en un campo tan próximo a nosotros como es el de la formación permanente; nos moveremos, por tanto, en el ámbito del diseño, aplicación y desarrollo de instrumentos y estrategias, técnicas y pedagógicas, a implementar en un colectivo -profesores de educación de adultosque, por el beneficio que proporciona la tecnología del momento, puede quedar inmerso en tiempo real en un proceso auténtico de investigación-acción; y todo ello, por tanto, sin perjuicio de que termine redundando en el incremento del conocimiento. Esta dinámica de funcionamiento se ajusta perfectamente, en nuestra opinión, a un enfoque, orientación o perspectiva reiteradamente debatido en nuestra comunidad científica -no hacemos explícita su denominación porque el contexto en el que se mueve nuestra reflexión invitaría a confundir definitivamente las cosas. Y, puesto que hemos dicho que nuestra intención no era reflexionar sobre nuevas o viejas tendencias y corrientes educativas, no ampliamos más esta primera reflexión que acabamos de hacer a propósito de nuestro trabajo, aunque sí nos interesaba dejar constancia de los supuestos y orientaciones científicas de las que partimos.

2.- Ya hemos señalado que nuestro grupo de estudio y aplicación del programa está formado por profesionales de la educación de adultos. Conviene en este punto exponer algunas de las razones que fundamentan esta decisión, para lo que resulta imprescindible partir del reconocimiento de algunos hechos; en nuestra opinión, se trata de uno de los colectivos profesionales de la educación más necesitados, al tiempo que susceptibles, de introducción de cambios y aplicación de innovaciones en la enseñanza. Así lo hemos podido comprobar en nuestros contactos con estos profesionales; quizás el propio ámbito sociocultural en el que se desenvuelven profesionalmente o la misma tipificación y consideración generalizada de este sector educativo se conviertan en acicates para su receptividad a planteamientos y actividades diferentes. Y no olvidamos el efecto multiplicador y los valores añadidos que se consiguen al 
intervenir sobre los profesionales que actúan con personas adultas; pero vayamos a los hechos que fundamentan nuestras razones.

De un lado, la formación inicial del profesorado de educación de adultos sólo tiene referencias muy generales a las características del aprendizaje propio de las personas adultas, a las particularidades de la organización curricular de los centros de educación de adultos y a los contextos de aislamiento geográfico y marginación sociocultural en los que habitualmente trabajan, con lo que estos profesionales frecuentemente acceden al ejercicio de su actividad en condiciones que de ninguna manera pueden calificarse de óptimas. En este caso, como en otros muchos, la distancia entre el tipo, orientación y características de la formación teóricopráctica inicial recibida y la realidad social y cultural de los entornos en los que normalmente desarrollan su actividad, así como las exigencias psicopedagógicas que se derivan de la tipología de alumno sobre el que se centra su actividad profesional, es muy grande. Las dificultades curriculares, metodológicas, motivacionales...con las que se encuentran son numerosas.

De otro lado, los medios de formación con los que cuentan los profesores en los centros de educación de adultos no siempre responden a las necesidades reales que manifestan dichos profesores y es obvio que no siempre existe un marco adecuado donde una necesidad de formación sentida y manifiestada pueda ser respondida en tiempo real. La situación más habitual viene dada por la ausencia de una red de interacción y comunicación con el objetivo específico de responder a las necesidades de formación del profesorado de este tipo de centros.

Con mucha frecuencia, la situación descrita anteriormente se debe a la distancia física entre los centros de educación de adultos y los lugares en los que existen Centros de Profesores, grupos de especialistas, bibliotecas especializadas e incluso otros profesores de la misma especialidad o interés. No debe ignorarse, para entender mejor la reflexión que venimos haciendo, que en general las mayores necesidades de educación permanente de adultos se corresponden con zonas de distribución geográfica muy diseminada o periférica de grandes ciudades y que los medios de formación más cualificados se encuentran concentrados en grandes núcleos de población, con lo que las iniciativas de formación presencial requieren de traslados y disponibilidades de tiempo que las reducen a situaciones ocasionales y distanciadas en el tiempo, provocando interrupciones en la actividad profesional y aumentando los costos por unidad de programación, al tiempo que disminuye el efecto multiplicador de la iniciativa así como el carácter de individualización y personalización de las situaciones.

En definitiva, se aprecia una serie de circunstancias que inclinan a pensar que este profesorado padece un mayor aislamiento profesional que el de otros niveles; como si la marginación que se asocia a las necesidades de formación de personas adultas se correspondiera con la situación en la que tienen lugar las intervenciones profesionales en este ámbito. Entendemos que la acción que estamos presentando es un buen instrumento para paliar esas situaciones de desigualdad que se generan.

3.- En el otro orden de reflexión, la aplicación de nuevas tecnologías a este sector educativo, las consideraciones a realizar podrían ser muchísimas. Descartada, como ya hemos anunciado, una amplia justificación de su utilización, consideramos suficiente con resaltar lo que parece evidente: la posibilidad que ofrecen de satisfacer un amplio espectro de necesidades y características específicas que presentan los profesionales de la educación de adultos y que recogíamos en el epígrafe anterior. Y no consideramos una ventaja menor la oportunidad que proporcionan para el fomento de la cooperación entre investigadores y profesionales de la educación de adultos, de manera que sea factible la implementación y 
desarrollo de servicios de asesoramiento y tutoría. Hemos de admitir que cada vez será más frecuente la presencia de medios tecnológicos en los centros de educación de adultos y que resulta evidente el potencial de aprovechamiento de dichos medios para la formación permanente de este profesorado, así como las posibilidades inmediatas y futuras que a través de los mismos se pueden ya poner en marcha, y todo ello en tiempo real y a bajo coste. Solamente nos gustaría añadir en este punto un aspecto: un interés no menor de este programa viene dado por su intención manifiesta -más aún, imperiosa necesidad, como veremos posteriormente- de poner en relación profesionales de la educación de adultos pertenecientes a varios países a la vez que establece puentes de conexión entre investigadores, especialistas y profesionales de la educación de adultos, también en este caso procedentes de distintos países; es una manera, sin duda, de construir la Europa de la Educación y la Cultura, de buscar y fomentar la dimensión europea de la educación, en definitiva, de construir lo que desde hace un tiempo nos viene ocupando, la Unión Europea.

4.- Una vez enunciados algunos supuestos básicos y motivada razonablemente la oportunidad, conveniencia y hasta necesidad de un programa de este tipo, procede que nos introduzcamos de lleno en la estructura y funcionamiento del programa, comenzando por hacer más explícitos su orientación y propósitos.

El programa pretende diseñar, implementar y aplicar lo que denominamos SISTEMA TUTOR INTERACTIVO destinado a la formación permanente de los profesores de educación de adultos. Se denomina TUTOR porque uno de los componentes del sistema -los profesores de educación de adultos- se toman como sujetos para iniciativas de formación permanente, mientras el resto de los componentes del sistema colabora en la orientación real o simulada de iniciativas de intervención en educación de adultos, de forma que, apoyándonos en las posibilidades interactivas de las nuevas tecnologías, se establezca una red de formación entre investigadores y profesionales de este campo para la promoción de la reflexión conjunta sobre las situaciones y acciones educativas específicas. El programa, por tanto, se concibe concretamente como un centro cooperativo de formación, un centro virtual de formación, una red de formación que, con base en la interacción que permiten las Nuevas Tecnologías de la Comunicación y de la Información, promueva la reflexión cooperativa sobre la situación y acción educativa específica en que se encuentran los profesionales de la educación permanente de adultos.

5.- La acción del sistema, como ya se ha señalado, recae, sobre profesorado de educación de adultos, entendiendo por tales los profesionales que desarrollan su actividad dentro de centros especializados de educación de adultos -más adelante haremos algunas precisiones en torno al grado o nivel de este sector que constituye el objeto de nuestro trabajo. El ámbito de las iniciativas de formación a desarrollar tiene relación con aspectos del aprendizaje de personas adultas dentro de metas del Sistema de Educación Básica; se excluyen, por motivos de precisión del programa, los ámbitos de alfabetización propiamente dicha y los de formación profesional así como los niveles secundarios y universitarios. Expresamente nos concentramos en profesores y actividades relacionadas con el nivel de formación básica, correspondiente al período de escolaridad obligatoria, dentro de la interpretación de cada país participante.

Hablamos de formación permanente porque:

- No pretendemos programar un curso con principio y fin, sino crear una estructura de interacción entre agentes de ese campo profesional.

- No pretendemos profundizar en un campo científico determinado relacionado con el 
proceso de enseñanza, sino promover un proceso sistematizado de reflexión, estudio, toma de decisiones y evaluación de resultados sobre acontecimientos del proceso de acción real considerados relevantes por los propios profesores.

- Se configura y se lleva a cabo respetando al máximo la situación del profesorado; se desarrolla en sus propios lugares de trabajo, dentro de sus disponibilidades de tiempo, y promueve un modo de interacción entre los agentes implicados en el que cada uno de ellos aporta y ejercita competencias que corresponden a sus propios compromisos y decisiones profesionales.

6.- Puesto que en nuestro trabajo se toma como elemento nuclear la reflexión sobre la situación y acción pedagógica del profesor de educación de adultos, las actividades iniciales a desarrollar y en las que nos encontramos inmersos son de dos tipos:

- Diseño de la estructura material y técnica de la red de telecomunicación, de forma que permita distintos niveles de acción y cooperación -más adelante volveremos sobre este punto.

- Determinación de las unidades básicas de formación, que se concretan a partir de la identificación, descripción y análisis de una situación. Puesto que en nuestra propuesta de sistema de formación permanente del profesorado de educación de personas adultas se toma como criterio general la reflexión sobre la actividad pedagógica del propio profesor, la unidad de formación será el análisis de una situación.

7.- Lo que acabamos de denominar 'unidades básicas de formación' constituye el punto de apoyo y elemento nuclear del programa. Su origen está en la realidad de la actividad pedagógica del profesional y su configuración se apoya en las dificultades o problemas que encuentra en situaciones educativas concretas; congruentes con el enfoque que presentábamos en el primer epígrafe, nuestro programa parte de casos reales procedentes del ejercicio cotidiano de los profesionales. Por tanto, las situaciones objeto de análisis y tratamiento pedagógico en la red son propuestas por los propios profesores de los centros de educación de adultos. Cada país participante está planteando en estos momentos un conjunto de situaciones y problemas que se vierten a la red, como paso previo para la determinación conjunta de las unidades de formación que van a ser utilizadas; se han exluido por razones obvias los aspectos y situaciones de tipo administrativo y político, incluyéndose solamente aspectos psicopedagógicos. Además, y puesto que una situación bien delimitada y relevante puede desarrollarse indefinidamente, nuestra actuación está sujeta a unos límites, definidos en términos de tratamiento de una unidad por trimestre, de manera que sean tres las unidades de análisis que constituyan el proceso de formación de profesores durante el año de aplicación del programa.

¿Qué estamos consiguiendo ya con nuestro programa y dinámica de funcionamiento?. Dado que nuestro énfasis recae sobre formación permanente en sujetos en ejercicio como formadores de personas adultas, queremos resaltar aquí el logro de un resultado que ya estamos obteniendo y que es, sin duda, de gran interés: la promoción de la reflexión de los propios actores sobre sus propias actuaciones. Dicho de otra manera, la secuencia de formación persigue dos objetivos: no sólo mejorar el repertorio de estrategias de intervención y enriquecer los conocimientos disponibles por los actores, sino también aumentar los niveles de metacognición del sujeto sobre sus propios procesos de actuación y de formación; éste último se ha puesto en marcha ya con la propia participación de los profesores en las diferentes sesiones de trabajo del programa. Y todo ello, al tiempo que creamos orientación y planificación heurística del plan autónomo-asistido de formación permanente en los sujetos. 
Ahora se entenderá mejor que el plan de formación que se experimenta, en vez de organizarse en función de contenidos, se estructure en torno a situaciones reales vividas como problema por parte de los educadores de personas adultas.

8.- En el epígrafe número seis aludíamos a la estructura técnica de la red y a los distintos niveles de acción y cooperación que debe permitir. Sin entrar en los pormenores técnicos, he aquí una relación mínima de acciones previstas:

- Discusión regional a propósito de la identificación de las situaciones de análisis.

- Propuesta y debate a nivel internacional.

- Discusión internacional entre los investigadores de la red a propósito del tratamiento pedagógico de las situaciones planteadas: aportación de experiencias, información sobre investigaciones, sugerencias bibliográficas, técnicas específicas.

- Constitución de grupos internacionales de trabajo sobre aspectos particulares de la situación.

- Consultorio a especialistas internacionales y debate de aportaciones.

- Servicio de S.O.S: buzón de consultas personales urgentes en relación a circunstancias puntuales de acciones formativas cotidianas, fuera o independientemente de las unidades de análisis.

9.- En función de los dos puntos anteriores, nuestro plan de trabajo viene desarrollándose de acuerdo a las siguientes fases:

- Primera fase: Toma de decisión en torno al número, tipo y características de las situaciones que constituyen las unidades de análisis de formación de los profesores de adultos. Esta actividad parte del trabajo y reflexión de los profesores de los centros de educación de adultos, pero también implica discusión a nivel regional e internacional entre los profesores y los expertos para consensuar cooperativamente las situaciones de análisis, de manera que quedan implicados todos los miembros del proyecto.

- Segunda fase: Toma de decisión en torno a la determinación de las actividades específicas a desarrollar para el tratamiento pedagógico y didáctico de las situaciones propuestas. Esta actividad implica fundamentalmente a los expertos en Pedagogía.

- Tercera fase: Vertido a la red internacional de las situaciones propuestas, puesta a punto de las actividades pedagógicas diseñadas y aplicación por parte de los profesores de los centros de educación de adultos; ésta fase requiere una dinámica interactiva y cooperativa entre todos los miembros del programa.

- Cuarta fase: Evaluación del funcionamiento de la red y de los resultados obtenidos en cuanto a grado de satisfacción del profesor, conocimientos y actitudes adquiridas y eficacia en la práctica educativa.

10.- Reiteradamente venimos haciendo alusión a la estructura geográfica de la red de formación; es el momento de hacerla explícita. La red está formada por investigadores y profesionales de la educación de adultos pertenecientes a tres regiones: Aveiro (Portugal), Castilla y León (España) y Liguria (Italia). En las tres regiones se dan circunstancias 
análogas: existe educación de adultos con programación regional-nacional, se trata de población diseminada y centros de educación de personas adultas en poblaciones pequeñas con dotación de profesores que intervienen de manera especializada y dedicación completa en este ámbito; además, las tres regiones cuentan con un organismo o institución que realiza investigaciones en el campo de la formación de personas adultas. Los profesores de los centros de educación de adultos constituyen el grupo de experimentación de la red; concretamente el número de centros y profesores participantes son los siguientes: Castilla y León: Palencia: 4 centros, 2 profesores por centro; Salamanca: 3 centros, 2 profesores por centro; Aveiro: 2 centros, 2 profesores por centro; Liguria: 2 centros, 3 profesores por centro.

11.- Una dificultad sobrevenida del epígrafe anterior nos ha llevado a concretar más el nivel de educación de adultos y, en consecuencia, perfil del profesor afectado en cada país, pues los tres países participantes presentan distinta estructura y funcionamiento de la educación de adultos. Tras un estudio de la educación de adultos en cada país, hemos llegado a detectar un nivel de convergencia entre los tres que constituirá nuestra zona de estudio y aplicación. En España, el ámbito considerado es el que comprende desde el nivel 2 de enseñanza básica hasta la ESPA (Educación Secundaria para Adultos), coincidente con la Educación básica obligatoria. En Portugal este período correspondería, por sus características y el perfil del profesional, al $2^{\circ}$ ciclo de la educación reglada. En Italia se considera que el tramo educativo que puede ser incluido, dadas las características del proyecto, es el que corresponde a la denominada Educación de Base.

Otras muchas incidencias podríamos relatar, como las derivadas del distinto nivel de tecnología que presentan los centros de adultos de las tres regiones intervinientes o simplemente las que se derivan de la presencia de tres idiomas; precisamente en este último aspecto venimos manteniendo como principio el desarrollo del trabajo simultáneamente en los tres idiomas, por entender que este hecho constituye un valor añadido de nuestro acción.

12.- Por último, como es obvio, todavía no podemos hablar de resultados; está previsto llevar a cabo una evaluación del funcionamiento de la red que incluya cuatro aspectos: grado de satisfacción del profesor, conocimientos y actitudes adquiridas, eficacia en la práctica educativa y nivel de interés demostrado por otros colectivos de profesores no implicados directamente en el proyecto. No obstante, recordemos lo que hemos apuntado en varios epígrafes anteriores: no puede considerarse un resultado menor, en nuestro tiempo y de cara al futuro, ni la reflexión de los mismos profesionales de la educación sobre sus propias intervenciones educativas ni el mismo hecho de la colaboración entre profesionales de regiones y países diferentes; y estas dos cosas ya las estamos consiguiendo.

Terminamos con la siguiente reflexión. Con mucha frecuencia se escuchan en la comunidad educativa diatribas varias sobre los efectos perniciosos de las nuevas tecnologías, concretamente sobre el aislamiento que provocan en el sujeto. No debemos olvidar, sin embargo, la característica-oportunidad que estas tecnologías ofrecen para la participación, colaboración...en la construcción de nuevos modos, situaciones y contextos. Somos conscientes de que estas tecnologías, sus redes y marañas no son precisamente una cooperativa, pero no todos los días nos levantamos con instrumentos en la mano que permitan la colaboración. ¿Podremos observar en el futuro cambios de mentalidad a partir de la conciencia de que la realidad y su diversidad, empezando por el mismo yo, se construye en base a tareas de desarrollo colectivo que trascienden el ámbito de los grupos primarios?. 
(C) Ediciones Universidad de Salamanca. 